\title{
Evaluación de 1.497 adultos mayores institucionalizados, usando el «sistema de clasificación de pacientes RU G T-18»
}

\author{
Pedro Paulo Marín $L^{1}$, Trinidad H oyl $M^{1}$, H omero Gac $E^{1}$, \\ Marcela Carrasco ${ }^{1}$, Patricio D uery Da, Kristina Petersen Ca, \\ Mauricio Cabezas $U^{a}$, Catalina D ussaillant $\mathrm{L}^{\mathrm{b}}$, Sergio Castro $\mathrm{H}^{1}$. \\ Assessment of 1497 Chilean nursing \\ home residents, using the Resource \\ Utilization Group method, RUG T-18
}

Background: There is little information about Chilean elderly residents of long term care facilities, regarding their characteristics and need for resources. Aim: To describe main characteristics and resource utilization of residents of one of the largest nursing homes in Chile, Fundación Las Rosas de Ayuda Fraterna. Material and Methods: In a cross sectional and descriptive study, all residents were evaluated using the RUG T-18 method, that assess activities of daily living and the complexity of their clinical situation. Results: We assessed 1497 subjects 60 years old and over (73\% women), with an age range of 60-106 years. Thirty six percent had urinary incontinence, 19\% required assistance for feeding, and 38\% needed help for walking or moving. Fifty seven percent were in the lowest category of complexity, «nstitutionalization». Very few residents were in the most demanding categories, no one classified as «ehabilitation», and only $0.7 \%$ were in «pecial Care». Conclusions: This study is an important start point to learn more about elderly subjects living in nursing homes in Chile (Rev Méd Chile 2004; 132: 701-6).

(Key Words: Aged; Housing for the elderly; Institutionalization)

Recibido el 17 de noviembre, 2003. Aceptado en versión corregida el 20 abril, 2004.

${ }^{1}$ Programa de Geriatría y Gerontología, Departamento de Medicina Interna, Facultad de Medicina, Pontificia Universidad Católica de Chile, Santiago de Chile.

anterno de Medicina, Facultad de Medicina, Pontificia Universidad Católica de Chile.

bAlumna de Medicina, Facultad de Medicina, Pontificia Universidad Católica de Chile.

Correspondencia a: Dr. Pedro Paulo Marín L. Departamento de Medicina Interna, Programa de Geriatría y Gerontología, Facultad de Medicina, Pontificia Universidad Católica de Chile. Casilla 114-D, Santiago Chile. E-mail: ppmarin@med.puc.cl 
L a población mundial está envejeciendo y Chile es parte de esta realidad. Según el CENSO de 2002, $11,4 \%$ de la población total $(1.717 .478$ chilenos) son adultos mayores de 60 años (AM), lo que corresponde a 412.000 personas más que hace diez años, siendo también el grupo que proporcionalmente más ha crecido anualmente $3,3 \%$ vs $2,1 \%$ de crecimiento en la población general $^{1}$. Este cambio en la sociedad chilena, plantea nuevos desafíos en salud pública, uno de ellos es el adecuado manejo de los AM que requieren «cuidados continuos (crónicos) o de largo plazo ${ }^{2}$.

Dichos cuidados se pueden realizar en la vivienda propia o en instituciones. Lo observado en los países más desarrollados, es que la mayoría de los AM pretenden permanecer en sus propias viviendas, para lo cual se han desarrollado programas de apoyo para ellos y sus familiares, con la intención de evitar o retardar el ingreso a una institución de larga estadía». A pesar de lo anterior, este tipo de cuidado institucional es una realidad y su número se incrementa cada año en todos los países ${ }^{3}$. En Estados Unidos de Norteamérica (USA), el $5 \%$ de los $\mathrm{AM}$ vive en una institución de cuidados prolongados; si se analiza las mujeres mayores de 85 años, el porcentaje sube a $25 \%$. Los usuarios de estos centros son principalmente mujeres, viudas, con discapacidad funcional, un tercio son mayores de 85 años y la mayoría tiene demencia o trastornos conductuales, incontinencia urinaria o requieren ayuda en las actividades de la vida diaria (AVD) 3,4 .

En Chile, se desconoce el número de $\mathrm{AM}$ que vive en instituciones ( hogares o casas de reposo»). Tampoco hay publicaciones respecto a las características de los AM institucionalizados, su nivel de fragilidad, el nivel de apoyo que requieren ni el tipo de servicio que reciben. Los escasos datos que existen son estudios realizados por nuestro grupo: el primero comparó nonagenarios de la comunidad versus institucionalizados y reveló que los últimos no eran más frágiles desde el punto de vista de salud, pero sí contaban con menor apoyo familiar, social y económico ${ }^{5}$; el segundo estudio fue realizado con pacientes ambulatorios, encontrándose que $7 \%$ no contaba con apoyo en caso de requerir cuidados prolongados; además, demostró un aumento sostenido de la fragilidad con mayor edad, una mayor necesidad de apoyo en AVD y mayor deterioro cognitivo $^{6}$, hechos que podrían relacionarse con mayor riesgo de ingresar a una institución de larga estancia.

Uno de los problemas del cuidado institucional, es el alto costo que implica atender a una población de AM que es cada vez más frágil y con mayores limitaciones y dependencia. El costo del cuidado en instituciones va a depender, en gran medida, del tipo de AM que se atienda y de la calidad de atención que se desee entregar. En USA, desde la década 1980-89, se ha analizado la forma de optimizar recursos económicos y humanos para disminuir los costos de salud, intentando mantener un alto estándar de atención. Así surgió el diseño de los ssistemas de clasificación de pacientes»(case-mix). El concepto se basa en que a pesar de que cada paciente es único, hay características comunes entre los sujetos ingresados que determinan el nivel de servicios que requerirán y por lo tanto su costo puede ser predecible. Se trata de estimar las necesidades reales de cada paciente (tipo de personal, tiempo de dedicación, etc), considerando su edad, situación clínica, diagnóstico clínico y el estado funcional en el diario vivir 7,8 .

Existen dos grandes grupos de sistemas de clasificación de pacientes, los que dependen del diagnóstico, denominados «isease Related Groups - DRGs»y los que son independientes del diagnóstico, llamados «Resources Utilization Groups - RUGs»?.

Los DRGs, en general, se centran en la utilización de recursos, pero por sus características son mejores en pacientes agudos y poco útiles para unidades que manejan pacientes de larga estancia o de cuidados continuos. En cambio, los RUGs, son un sistema que se ajusta mejor a la población AM institucionalizada, siendo la versión RUG T-18 corta, más fácil de emplear y clasifica a los pacientes en función de dos dimensiones: la autonomía personal y la complejidad clínica, de manera que cada categoría representa un nivel de consumo de recursos diferentes y jerárquicos. Si el paciente clasifica para una categoría más compleja, mayor será la cantidad de recursos utilizados en él y viceversa. El sistema RUG T-18 ha sido validado internacionalmente para ser usado en el contexto de residencias de larga estancia de $\mathrm{AM}^{7-9}$ y en Chile 
fue ya usado por nuestro grupo en AM hospitalizados $^{10}$. Este instrumento es útil, tanto para describir mejor una población de AM, como para optimizar los sistemas administrativos de admisión del paciente o el tipo, tiempo y dedicación del personal requerido, y los otros recursos económicos, con el fin de lograr una mejor atención al paciente ${ }^{7-10}$

En Chile no existe un catastro nacional de los hogares de larga estancia, ni se conoce el número total de AM institucionalizados, menos aún se conocen las características de ellos, su nivel de dependencia ni de fragilidad. Por ello, nuestro grupo realizó el presente trabajo con el objetivo de evaluar a un grupo grande de AM institucionalizados de escasos recursos, aplicando el sistema de clasificación de pacientes RUG T-18.

\section{Material y MÉTOdo}

Durante el mes de octubre de 2002, se realizó una evaluación transversal, a todas las personas institucionalizados en las sedes de la «Fundación Las Rosas de Ayuda Fraterna»de la Región Metropolitana. Dicha evaluación, fue realizada por los miembros del equipo de geriatría, internos y alumnos de la Escuela de Medicina de la Pontificia Universidad Católica de Chile y por el personal de la Fundación Las Rosas. Todos los evaluadores fueron previamente capacitados en la aplicación del instrumento RUG T-18. Se evaluó a los AM y en caso que ellos no se encontraran en condiciones de responder, se obtuvo la información de los cuidadores. Los AM que participaron, lo hicieron con consentimiento informado verbal.

Se utilizó como método de evaluación el «istema de Clasificación de Pacientes RUG T$18{ }^{8,10}$, que consta de dos partes: la primera evalúa las actividades de la vida diaria (AVD), calculándose un índice con las puntuaciones otorgadas en cada una de las siguientes tres actividades consideradas: continencia urinaria (con tres niveles), alimentación (con cuatro niveles) y deambulación (con tres niveles). Al sumar los puntajes de cada categoría se obtiene el puntaje de AVD total.

La segunda parte del RUG T-18, clasifica a los pacientes según la «omplejidad de su Situación
Clínica» y establece cinco categorías jerárquicas. Cuando un paciente reúne al menos un criterio para ser incluido en una categoría de rango superior (mayor complejidad), se le considera en esa categoría y no es necesario buscar criterios de inclusión en el resto de las categorías subyacentes. La jerarquía entre categorías se establece en el siguiente orden (de superior a inferior): rehabilitación, cuidados especiales, clínicamente complejos, alteraciones de conducta y funciones físicas reducidas (ver instrumento completo en referencia 10).

Para el análisis estadístico se utilizó el programa SPSS. Para la comparación de promedios se usó el test $t$ de Student para variables independientes, y para la comparación de porcentajes, el test de Chi cuadrado. Se consideró significativo un valor $\mathrm{p}<0,05$.

\section{RESULTADOS}

De los 1.550 sujetos posibles, fueron seleccionados para el presente estudio aquellos mayores de 60 años cuya evaluación estaba completa, dando un total de 1.497 adultos mayores (97\% de la población total de AM residentes en hogares de la Fundación Las Rosas en la Región Metropolitana). El promedio de edad fue 81 años (rango entre 60 y 106 años), 77,8\% presentaban una edad mayor 0 igual a 75 años y $73 \%$ eran mujeres $(n=1.096)$. Las mujeres, tenían una edad promedio significativamente mayor: $82,6 \pm 8,1$ años versus $76,8 \pm 7,8$ años en hombres $(p<0,05)$.

En la Tabla 1 se muestran los resultados obtenidos con el «istema de clasificación RUG T18». Al analizar las actividades de la vida diaria (AVD), destaca que más de un tercio $(36,2 \%)$ del total de los pacientes presentaba incontinencia urinaria, de los cuales sólo 1\% se encontraba en programas de reentrenamiento vesical. En el grupo etario $\geq 75$ años, los sujetos incontinentes alcanzaron un porcentaje significativamente mayor $(39 \%$ versus $27 \%, p<0,01)$. En cuanto a alimentación, la gran mayoría, (81\% del total) fue considerado autosuficiente para AVD y sólo $12 \%$ era totalmente dependiente para alimentarse, sin observarse diferencias significativas por edad. Con respecto a la movilidad, $62 \%$ de los AM eran independientes, pero observamos diferencias es- 
Tabla 1. Clasificación de adultos mayores institucionalizados con RU G -T 18: resultados de las Actividades de la Vida D iaria, según edad

\begin{tabular}{|c|c|c|c|c|}
\hline $\begin{array}{l}\text { Actividades de la } \\
\text { vida diaria (AVD) }\end{array}$ & Total & $\begin{array}{l}<75 \text { años } \\
\mathrm{n}=1.497\end{array}$ & $\begin{array}{c}\geq 75 \text { años } \\
n=332\end{array}$ & $\begin{array}{l}\text { Valor } \mathrm{p} \\
\mathrm{n}=1.165\end{array}$ \\
\hline \multicolumn{5}{|l|}{ Continencia } \\
\hline Autosuficiente & $63,1 \%$ & $72,6 \%$ & $60,4 \%$ & $<0,001$ \\
\hline Requiere ayuda & $35,1 \%$ & $26,5 \%$ & $38,4 \%$ & $<0,001$ \\
\hline En reentrenamiento vesical & $1,1 \%$ & $0,9 \%$ & $1,2 \%$ & $<0,001$ \\
\hline \multicolumn{5}{|l|}{ Alimentación } \\
\hline Autosuficiente & $81 \%$ & $83,7 \%$ & $80,3 \%$ & 0,153 \\
\hline Requiere ayuda continuamente & $6,9 \%$ & $6,3 \%$ & $7,1 \%$ & 0,613 \\
\hline Completamente dependiente & $11,6 \%$ & $9,3 \%$ & $12,3 \%$ & 0,140 \\
\hline Con SNG-parenteral & $0,4 \%$ & $0,6 \%$ & $0,3 \%$ & 0,509 \\
\hline \multicolumn{5}{|l|}{ Movilidad } \\
\hline Independiente & $62,3 \%$ & $72,3 \%$ & $59,5 \%$ & $<0,001$ \\
\hline Ayuda de 1 persona & $19,3 \%$ & $12,7 \%$ & $21,2 \%$ & $<0,001$ \\
\hline Ayuda de 2 o más personas & $18,4 \%$ & $15,1 \%$ & $19,3 \%$ & 0,077 \\
\hline Indice AVD (promedio \pm DS) & $4,26 \pm 1,7$ & $3,98 \pm 1,6$ & $4,34 \pm 1,7$ & \\
\hline
\end{tabular}

Tabla 2. Clasificación de adultos mayores institucionalizados con RU G -T 18: según las categorías jerárquicas y por edad

\begin{tabular}{|c|c|c|c|c|}
\hline $\begin{array}{l}\text { Categoría RUG-T18 } \\
\text { (de mayor a menor) }\end{array}$ & $\begin{array}{c}\text { Total } \\
\mathrm{n}=1.497(\%)\end{array}$ & $\begin{array}{c}<75 \text { años } \\
\mathrm{n}=332(\%)\end{array}$ & $\begin{array}{c}\geq 75 \text { años } \\
\mathrm{n}=1.165(\%)\end{array}$ & $\mathrm{p}$ \\
\hline Rehabilitación & $0 \quad(0 \%)$ & & & \\
\hline Cuidados especiales & $11 \quad(0,7 \%)$ & $2 \quad(0,6 \%)$ & $9(0,8 \%)$ & ns \\
\hline Clínicamente complejos & 195 (13\%) & $55 \quad(16,6 \%)$ & $140(12,0 \%)$ & ns \\
\hline Alteraciones de conducta & $438(29,3 \%)$ & $(27,4 \%)$ & $347(29,8 \%)$ & ns \\
\hline Institucionalizado & $853 \quad(57 \%)$ & $(55,4 \%)$ & $669(57,4 \%)$ & ns \\
\hline
\end{tabular}

tadísticamente significativas por edad; en los menores de 75 años, $72 \%$ era independiente, mientras que en $l o s \geq 75$ años este porcentaje sólo alcanza a $60 \%(\mathrm{p}<0,001)$, siendo más frecuente la necesidad de ayuda de una persona (21\%) o de dos o más personas (19\%). El promedio de puntaje asignado al índice AVD fue de 4,26 $\pm 1,7$ sin diferencias significativas entre los grupos etáreos.

La distribución de los AM institucionalizados según las Categorías Clínicas del RUG, puede verse en la Tabla 2. Más de la mitad de los AM (57\%) clasificaron en la categoría Institucionaliza- dos, es decir, la de menor complejidad médica y menor nivel de utilización de recursos. En el otro extremo, no hubo pacientes que cumplieran los criterios para clasificar en la categoría de rehabilitación, ya que se exige que el paciente reciba un mínimo de media hora diaria de terapia, y no había pacientes con esta intensidad de tratamiento. Por otro lado, sólo 11 sujetos $(0,7 \%)$ clasificaron en Cuidados Especiales, la segunda categoría de mayor necesidad de recursos humanos y de alta complejidad. No se observó diferencias estadísticamente significativas al analizar por edad. Al compararlos por sexo, se observó que existen 
diferencias significativas, ya que entre los hombres hubo un mayor porcentaje de pacientes en el grupo de clínicamente complejos (20,9\% hombres versus $10,1 \%$ mujeres, $p<0,001$ ), la tercera categoría en complejidad, mientras que las mujeres se encontraron más en la categoría inmediatamente inferior, alteraciones de conducta $(33,4 \%$ mujeres versus $18 \%$ hombres, $\mathrm{p}<0,001$ ).

\section{DisCUSIÓN}

El incremento de la población adulta mayor (AM) en Chile es un hecho de gran importancia a nivel de salud pública ${ }^{2}$. Según datos recientemente publicados, se estima que en el año 2010 existirán 50 AM por cada 100 menores de 15 años y si las tendencias se mantienen, estas cifras se igualarían en el año 2034. La desventaja del envejecimiento poblacional, en países de desarrollo económico intermedio como Chile, es que los AM se ven afectados fuertemente por la pobreza. Según la encuesta CASEN de MDEPLAN año 2000, 8\% de los AM son pobres, siendo $1,6 \%$ indigentes ${ }^{11}$. Se sabe que la pobreza es un factor de riesgo de institucionalización, que surge como una alternativa de cuidado y protección al AM frágil sin una red de apoyo suficiente ${ }^{3,4}$.

En Chile no existe un catastro nacional de los hogares de larga estancia ni se conoce el número total de AM institucionalizados, menos aún se conocen las características de ellos, su nivel de dependencia ni fragilidad, como tampoco si los hogares que los acogen cumplen con los requerimientos mínimos de cuidado. Dada esta realidad, nuestro grupo quiso conocer más a fondo la realidad de AM pobres institucionalizados en una de las principales fundaciones de beneficencia de la Región Metropolitana, Fundación Las Rosas de Ayuda Fraterna. Esta Fundación tiene criterios de ingreso para los AM, dentro de los que destaca que el AM debe ser de bajos ingresos (indigente o con jubilación que no le alcance a costear un hogar de ancianos privado), debe ser mayor de 60 años, valente o semi-valente, sin familiares que puedan acogerlo en forma adecuada, por lo que los resultados de este estudio no son necesariamente aplicables a otros centros.
Bajo este marco, se pueden analizar los resultados encontrados usando RUG T-18 como método de clasificación de los pacientes, método validado internacionalmente y utilizado previamente en la evaluación de AM tanto institucionalizados como en otros contextos ${ }^{8-10}$. Es importante destacar que la muestra es de una magnitud suficiente para darle validez a los resultados y extrapolarlos a poblaciones que compartan la mayoría de los criterios de ingreso descritos.

Destaca que el número de mujeres institucionalizadas es muy superior al de hombres, hallazgo que concuerda con lo descrito en la literatura ${ }^{11,12}$, así como también que ellas presenten un mayor promedio de edad, ya que se sabe que la edad es uno de los factores más importantes en la institucionalización. Las mujeres, además, tienen una expectativa de vida al nacer, mayor que los hombres, alcanzando en Chile 6 años más que los hombres (79 para las mujeres y 73 años para los hombres) ${ }^{11}$. Sin embargo, la edad no es la única razón que explica esta diferencia y están descritas otras causas, dentro de ellas, sociales, que podrían relacionarse con una mayor frecuencia de institucionalización femenina ${ }^{3,4}$.

Respecto a las actividades de la vida diaria, destaca que la incontinencia urinaria es un problema importante y las cifras son mayores que las descritas previamente por nuestro grupo de geriatría en AM que viven en la comunidad y en nonagenarios institucionalizados ${ }^{5,6}$. En alimentación la muestra es bastante autovalente. La funcionalidad de los sujetos estudiados, en cuanto a su capacidad de movilizarse, muestra que casi $40 \%$ de la población es dependiente, lo que implica un alto nivel de necesidad de recursos para una institución, tanto en personal de apoyo, como en infraestructura. Estos porcentajes son similares a los descritos por nosotros en otro estudio con pacientes hospitalizados, pero mucho más altos que encontrados en pacientes ambulatorios $\mathrm{s}^{6,10,13}$.

En suma, un número significativo de $\mathrm{AM}$ pobres institucionalizados se encuentra con importantes alteraciones funcionales por lo tanto con gran fragilidad, lo que es un factor de mayor costo de atención (humano y económico), incluso de mortalidad a un año plazo 3,14,15. Lo anterior tiene 
importantes consecuencias, ya que conlleva una necesidad de recursos especializados focalizados en este grupo de mayor fragilidad. Llama la atención que, aunque en los criterios de ingreso de la institución se les exige una ssemi-valencia o autovalencia», hayamos encontrado un alto porcentaje de dependientes. Esto puede explicarse, en parte, porque la mayoría de los AM que ingresan lo hacen en forma definitiva, por lo tanto, siguen envejeciendo en la institución y van desarrollando, con los años, enfermedades crónicas y distintas limitaciones y discapacidades que no tenían en el momento de ingresar.

\section{REFERENCIAS}

1. Censo de Población y Vivienda. Instituto NacioNAL DE ESTADísTiCA. 2002.

2. MaRín PP. La situación del adulto mayor en Chile (artículo especial). Rev Méd Chile 1998; 125: 1207-12.

3. Brosky J, Habib J, Hirschfeid M. Key Policy issues in Long Term Care. The cross-cluster initiative on longterm care, World Health Organization. WHO 2003.

4. HiRSChFELD M, LINDSEY E. Community Home-based care in resource-limited settings: a framework for action. WHO 2002.

5. Marín PP, Castro S, Galeb I, Valenzuela E, Hoyl T. Estudio comparativo de nonagenarios que viven en sus propios hogares versus los institucionalizados. Rev Méd Chile 1998; 126: 761-8.

6. Marín PP, Kornfeld R, Somiai E, Valenzuela E, CASTRO S. Valoración geriátrica ambulatoria de 2.116 adultos mayores pobres. Rev Méd Chile 1998; 126: 609-14.

7. FriEs BE, COONEY L Resource Utilization Groups: A patient classification system for long-term care. Medical Care 1985; 23: 110-22.

8. Fries B, Schneider D, Foley W, Dowling M. Casemix classification of medicare residents in skilled nursing facilities: Resource Utilization Groups (RUG-T18). Med Care 1989; 27: 843-58.

9. Carpenter Gi, Main A, Turner GF. Casemix for elderly inpatient: Resource Utilization Group (RUGs) validation project. Casemix for the inpatient Workin Group. Age Ageing 1995; 24: 5-13.
Debemos concluir que la población de AM pobres institucionalizados estudiados, es un grupo muy heterogéneo y más frágil que la población chilena ambulatoria de edades comparables. Este es el primer estudio de población de AM residentes en instituciones en nuestro país y entendemos que nuestros resultados son importantes para la planificación de estrategias de intervención, reordenamiento y asignación de recursos humanos y económicos, con el fin de mejorar la calidad de vida, poniendo énfasis en áreas como la prevención de discapacidad y rehabilitación, áreas que mejoran la funcionalidad del $\mathrm{AM}^{15}$.

10. Marín PP, Valenzuela E, Castro S, Rodríguez F, Mouna O, CubiLos A et al. Resultados al aplicar el sistema de clasificación de pacientes «RUG-T18» en ancianos ingresados a un hospital universitario. Rev Méd Chile 1998; 126: 761-8.

11. Perfil del Adulto Mayor en Chile. Desarrollando respuestas integradas de Sistemas de Cuidados de Salud para una población de rápido envejecimiento (INTRA). Publicación Ministerio de Salud y OPS-OMS, 2002.

12. Bird C, Shugarman L, Lynn J. Age and gender differences in health care utilization and spending for medicare beneficiaries in their last years of life. J Palliat Med 2002; 5: 705-12.

13. Marín PP, Valenzueia E, Saito N, Castro S, Hoyl T. Experiencia piloto en el uso de una ficha de evaluación geriátrica ambulatoria. Rev Méd Chile 1996; 124: 701-6.

14. Bean J, Kiely D, Cairns K, Morris J. Influence of poststroke urinary incontinence on disability: the nursing home setting. Am J Phys Med Rehabil 2003; 82: 175-81.

15. FLACKER J, KIELY D. Mortality-related factors and 1 year survival in nursing home residents. J Am Geriatr Soc 2003; 51: 213-21.

Agradecimiento

Los autores agradecen a los miembros del Directorio de Salud y al personal de la Fundación Las Rosas de Ayuda Fraterna, por el apoyo y cooperación en la realización de esta investigación. 